\title{
On the geodesic mappings of pseudo-Riemannian spaces with special supplementary tensor
}

\author{
V. Kiosak, O. Prishlyak, O. Lesechko
}

\begin{abstract}
The paper treats two pseudo-Riemannian spaces having common geodesic lines. Certain algebraic and differential conditions are imposed on the Riemann tensor of one of the spaces, while an operation of lowering indices and a calculation of the covariant derivative is carried out with respect to metrics and connection objects of the another space. In order to study the objects we introduce a special supplementary tensor. It is proven that, when the additional conditions are true, then either the spaces do not admit non-trivial mappings or the spaces are equidistant spaces.

We apply tensor methods without limitations imposed on the sign of the metric under question.
\end{abstract}

\begin{abstract}
Анотація. В роботі досліджуються два псевдоріманових простори, які мають спільні геодезичні лінії. Вимагається виконання умов алгебраїчного та диференціального характеру на тензор Рімана одного з них. А операція опускання індексів та обчислення коваріантної похідної здійснюється відносно метрики та об'єктів зв'язності іншого простору. Для досліджень використовується спеціальний допоміжний тензор. Доведено, що виконання додаткових умов приводить до просторів, що не допускають нетривіальних геодезичних відображень, або простори належать до еквідістантних просторів.

Використовуються тензорні методи без обмежень на знак метрики.
\end{abstract}

$U D C 514.765 .1+512.813 .4$

Keywords: pseudo-Riemannian spaces, geodesic mapping, supplementary tensor

Ключові слова: псевдоріманові простори, геодезичні відображення, допоміжний тензор

DOI: http://dx.doi.org/10.15673/tmgc.v14i4.2140 


\section{INTRODUCTION}

A long history of the theory of geodesic mappings made it into a classic branch of theory of pseudo-Riemannian spaces. It has rich applications in the general relativity theory and mechanics, and became a source of methods and new problems for other branches both of differential geometry and of topology, as well as of theory of differential equations and functional analysis $[6,22,23,26,28]$. Nowadays, the researchers work on the three problems posed over hundred years ago. Namely, they are:

(1) to establish whether a given pseudo-Riemannian space admits nontrivial geodesic mappings;

(2) to find every pseudo-Riemannian spaces which can be images of a given space in the course of non-trivial geodesic mapping;

(3) to clarify for a given pair of pseudo-Riemannian spaces whether a nontrivial geodesic mapping is possible between them.

Every above-mentioned problem can be solved by the available methods. However, the exact solution is often hampered by difficulties of a technical character. This peculiarity re-enforces the need to search for new methods and specific indicators, which could simplify the research. This work presents a new method of the latter type, namely we introduce a special supplementary tensor. This tensor links two pseudo-Riemannian spaces, which are in geodesical correspondence, and a vector defining the correspondence. The research on properties of special supplementary tensor permits to study some interesting properties of spaces admitting non-trivial mappings

\section{Preliminary notions}

A bijection between points of pseudo-Riemannian spaces $V_{n}$ with a metric tensor $g_{i j}$ and $\bar{V}_{n}$ with a metric tensor $\bar{g}_{i j}$ is called a geodesic mapping, whenever it sends every geodesic line of $V_{n}$ into a geodesic line of $\bar{V}_{n}$.

Pseudo-Riemannian spaces $V_{n}$ and $\bar{V}_{n}$ admitting a geodesic mapping between them are called either spaces in geodesic correspondence or spaces belonging to the same geodesic class.

The following equations present necessary and sufficient conditions [24] for existence of geodesic mappings between pseudo-Riemannian spaces $V_{n}$ and $\bar{V}_{n}$ :

$$
\bar{\Gamma}_{i j}^{h}=\Gamma_{i j}^{h}+\varphi_{i} \delta_{j}^{h}+\varphi_{j} \delta_{i}^{h} .
$$

Taking into account the covariant stability of the metric tensor we get

$$
\bar{g}_{i j, k}=2 \varphi_{k} \bar{g}_{i j}+\varphi_{i} \bar{g}_{j k}+\varphi_{j} \bar{g}_{i k},
$$


here $\varphi_{i}$ is some gradient (with a necessity) vector, $\Gamma_{i j}^{h}, \bar{\Gamma}_{i j}^{h}$ are Christoffel symbols $V_{n}$ and $\bar{V}_{n}$ respectively, $\delta_{i}^{h}$ are Kronecker symbols, and comma "," is a sign of a covariant derivative by the connection of $V_{n}$.

Equations (2.1) and (2.2) are equivalent to necessary and sufficient conditions to existene of the geodesic correspondence between two pseudoRiemannian spaces $V_{n}$ and $\bar{V}_{n}$. The system of equations (2.2) is called a non-linear form of the main equations of the theory of geodesic mappings.

For a geodesic mapping the necessary condition can be written down as follows:

$$
\begin{gathered}
\bar{R}_{i j k}^{h}=R_{i j k}^{h}+\varphi_{i j} \delta_{k}^{h}-\varphi_{i k} \delta_{j}^{h}, \\
\bar{R}_{i j}=R_{i j}+(n-1) \varphi_{i j},
\end{gathered}
$$

where $\varphi_{i j}=\varphi_{i, j}-\varphi_{i} \varphi_{j}$ and $R_{i j k}^{h}, R_{i j k}$ are Riemann and Ricci tensors respectively.

Non-homothetic geodesic mapping is called non-trivial. A mapping is non trivial when the following condition is true $\varphi_{i} \not \equiv 0$.

A pseudo-Riemannian space $V_{n}$ admits a non-trivial mapping if and only if the following system of differential equations has a solution in the given space

$$
a_{i j, k}=\lambda_{i} g_{j k}+\lambda_{j} g_{i k}
$$

with respect to the tensor $a_{i j}=a_{j i} \neq c g_{i j}$ and to the vector $\lambda_{i}=\lambda_{, i} \neq 0$. This system is called a linear form of main equations of the theory of geodesic mappings.

Differential extensions of linear forms of main equations of the theory of geodesic mappings can be written down as follows, see [24]:

$$
n \lambda_{i, j}=\mu g_{i j}+a_{\alpha i} R_{j}^{\alpha}-a_{\alpha \beta} R_{. i j}^{\alpha \beta},
$$

where $\mu=\lambda_{\alpha, \beta} g^{\alpha \beta}, R_{j}^{i}=R_{\alpha j} g^{\alpha i}, R^{h}{ }_{i j}{ }^{k}=R_{i j \alpha}^{h} g^{\alpha k}$, and $g^{i j}$ are elements of a matrix inverse to $g_{i j}$.

The latter implies that

$$
(n-1) \mu_{, i}=2(n+1) \lambda_{\alpha} R_{i}^{\alpha}+a_{\alpha \beta}\left(2 R_{. i,{ }_{i}{ }^{\beta}}^{\alpha}-R_{, i}^{\alpha \beta}\right),
$$

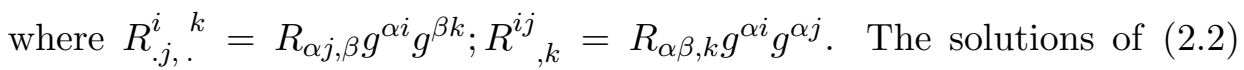
and (2.5) are related by the following formula

$$
\begin{aligned}
a_{i j} & =e^{2 \varphi} \bar{g}^{\alpha \beta} g_{\alpha i} g_{\beta j}, \\
\lambda_{i} & =-e^{2 \varphi} \bar{g}^{\alpha \beta} g_{\alpha i} \varphi_{\beta} .
\end{aligned}
$$

The system of equations (2.5), (2.6) and (2.7) opens a possibility to answer the following question in general: whether a given pseudo-Riemannian space $V_{n}$ admits a geodesic mapping onto a pseudo-Riemannian space $\bar{V}_{n}$. The 
question is reduced to the problem of integrability conditions for these equations and their differential extensions. The principal achievements in the theory of geodesic mappings of pseudo-Riemannian spaces are related with investigations of the linear form of the main equations. Vice versa, non-linear forms allow to obtain new results as demonstrated in $[1,8,11]$.

\section{Supplementary tensor}

Integrability conditions of (2.2), taking into account the Ricci identity, can be transformed into the following

$$
\bar{g}_{\alpha i} R_{j k l}^{\alpha}+\bar{g}_{j \alpha} R_{i k l}^{\alpha}=\varphi_{l, i} \bar{g}_{j k}+\varphi_{l j} \bar{g}_{i k}-\varphi_{k i} \bar{g}_{j l}-\varphi_{k j} \bar{g}_{i l},
$$

where $R_{i j k}^{h}$ is Riemann tensor of $V_{n}$.

Grouping (3.1), we obtain

$$
\begin{aligned}
& \bar{g}_{\alpha i} R_{j k l}^{\alpha}+\left(\varphi_{k, j}-\varphi_{k} \varphi_{j}\right) \bar{g}_{i l}-\left(\varphi_{l, j}-\varphi_{l} \varphi_{j}\right) \bar{g}_{i k}+ \\
& +\bar{g}_{\alpha j} R_{i k l}^{\alpha}+\left(\varphi_{k, i}-\varphi_{k} \varphi_{i}\right) \bar{g}_{j l}-\left(\varphi_{l, i}-\varphi_{l} \varphi_{i}\right) \bar{g}_{j k}=0 .
\end{aligned}
$$

Taking into account the fact that $\bar{g}_{i j}=\bar{g}_{\alpha j} \delta_{i}^{\alpha}$ and the equation (2.4), we get the following expression

$$
\bar{g}_{\alpha i} D_{j k l}^{\alpha}+\bar{g}_{\alpha j} D_{i k l}^{\alpha}=0,
$$

where

$$
D_{j k l}^{h}=R_{j k l}^{h}+\delta_{l}^{h}\left(\varphi_{k, j}-\varphi_{k} \varphi_{j}\right)-\delta_{k}^{h}\left(\varphi_{l, j}-\varphi_{l} \varphi_{j}\right) .
$$

The tensor $D_{j k l}^{h}$ is called supplementary.

Let us note that a supplementary tensor is not an inner object, as far as it includes elements of a vector $\varphi_{i}$. On the other hand, it includes Riemann tensor, which is an inner object and complies to well-known algebraic conditions. A supplementary tensor is not only a Riemann tensor when the pseudo-Riemannian space admits non-trivial geodesic mappings. We will take into account only these spaces.

Taking into account (2.3) and (3.2) we can write down

$$
\bar{R}_{i j k}^{h}=D_{i j k}^{h} .
$$

This illustrates why algebraic conditions imposed on a tensor $D_{i j k}^{h}$ are equivalent to conditions on tensor $\bar{R}_{i j k}^{h}$. Moreover, differential conditions imposed on $D_{i j k}^{h}$ can be transformed, when taking into account (2.1), with respect to a choice of a connection, by which the covariant derivative is calculated. In particular, the following identities hold:

$$
\begin{gathered}
D_{i j k}^{h}+D_{i k j}^{h}=0, \quad D_{i j k}^{h}+D_{j k i}^{h}+D_{k i j}^{h}=0 \\
D_{j k l, i}^{h}+D_{j l i, k}^{h}+D_{j i k, l}^{h}=\delta_{i}^{h} \varphi_{\alpha} R_{j l k}^{\alpha}+\delta_{k}^{h} \varphi_{\alpha} R_{j i l}^{\alpha}+\delta_{l}^{h} \varphi_{\alpha} R_{j k i}^{\alpha} .
\end{gathered}
$$


The following conditions are true for the tensor $D_{h i j k}=g_{\alpha h} D_{i j k}^{\alpha}$

$$
D_{h i j k}+D_{i h j k}=g_{h k} \varphi_{i j}-g_{h j} \varphi_{i k}+g_{i k} \varphi_{h j}-g_{i j} \varphi_{h k} .
$$

When the tensor $D_{h i j k}$ is skew-symmetric with respect to the first pair of indices in the pseudo-Riemannian space $V_{n}$, then

$$
g_{h k} \varphi_{i j}-g_{h j} \varphi_{i k}+g_{i k} \varphi_{h j}-g_{i j} \varphi_{h k}=0 .
$$

Wrapping by indices $h$ and $k$, we obtain

$$
\varphi_{i j}=\frac{\Delta_{2} \varphi-\Delta_{1} \varphi}{n} g_{i j},
$$

where $\Delta_{2} \varphi=\varphi_{\alpha, \beta} g^{\alpha \beta} ; \quad \Delta_{1} \varphi=\varphi_{\alpha} \varphi_{\beta} g^{\alpha \beta}$.

Substituting (3.5) into (3.4), we can see that this condition is sufficient for the tensor $D_{h i j k}$ to be skew-symmetric with respect to the first pair of indices. Thus we get the following theorem:

Theorem 3.1. The supplementary tensor $D_{\text {hijk }}$ is skew-symmetric with respect to the first pair of indices with a necessity and sufficiency, when the conditions (3.5) are true.

Equation (3.5) can be transformed to the following expression by substitution of $\psi=e^{-\varphi}$ :

$$
\psi_{, i j}=\rho g_{i j}
$$

where

$$
\rho=-e^{-\varphi} \frac{\Delta_{2} \varphi-\Delta_{1} \varphi}{n} .
$$

The vector field $\psi_{, i}$ corresponding to the equation (3.6), where $\rho$ is a certain invariant, is called a concircular vector field, and every pseudoRiemannian space $V_{n}$ in which there exists a concircular vector field is called an equidistant space, [24].

In the case $\rho \neq 0$ the space $V_{n}$ is called an equidistant space of a main type, while in the case $\rho=0$ it is an equidistant space of a special type.

Consider pseudo-Riemannian space $V_{n}$, in which the supplementary tensor $D_{i j k}^{h}$ corresponds to a condition analogous to the differential Bianchi identity, namely

$$
D_{i j k, m}^{h}+D_{i k m, j}^{h}+D_{i m j, k}^{h}=0 .
$$

Then, the expression (3.3) implies that

$$
\delta_{i}^{h} \varphi_{\alpha} R_{j l k}^{\alpha}+\delta_{k}^{h} \varphi_{\alpha} R_{j i l}^{\alpha}+\delta_{l}^{h} \varphi_{\alpha} R_{j k i}^{\alpha}=0 .
$$

Wrapping by indices $h$ and $i$, we get

$$
(n-2) \varphi_{\alpha} R_{j l k}^{\alpha}=0 .
$$


When $n \neq 2$, then the expression (3.8), together with (3.3), implies (3.7). In other words, the following theorem holds:

Theorem 3.2. A pseudo-Riemannian space $V_{n}(n>2)$ has a supplementary tensor conforming to the conditions (3.7) if and only if conditions (3.8) are satisfied.

The integrability conditions for equations (3.6) are the following:

$$
\psi_{\alpha} R_{i j k}^{\alpha}=\rho_{, k} g_{i j}-\rho_{, j} g_{i k} .
$$

Multiplying (3.9) by vector $\psi^{i}=\psi_{\alpha} g^{\alpha i}$ and wrapping by index $i$, we obtain

$$
\rho_{, k} \psi_{j}-\rho_{, j} \psi_{k}=0 \text {. }
$$

Therefore, as $\psi_{i} \neq 0$, we can choose a vector $\xi^{j}$ so that $\psi_{\alpha} \xi^{\alpha}=1$. Then it is easy to see that

$$
\rho_{, k}=B \psi_{k},
$$

where $B$ is some invariant, $B=\rho_{, \alpha} \xi^{\alpha}$.

Taking into account (3.10), one can rewrite (3.9) as follows

$$
\psi_{\alpha} R_{i j k}^{\alpha}=B\left(\psi_{k} g_{i j}-\psi_{j} g_{i k}\right) \text {. }
$$

Passing to the vector $\varphi_{i}$, we also get

$$
\varphi_{\alpha} R_{i j k}^{\alpha}=B\left(\varphi_{k} g_{i j}-\varphi_{j} g_{i k}\right) .
$$

Thus, when a supplementary tensor is skew-symmetric with respect to the first pair of indices, then the conditions (3.11) hold. Moreover, if the conditions (3.7) are also true, then $B=0$, whence (3.10) implies that $\rho_{, k}=0$ as well.

Thus we get the following corollary of Theorems 3.1 and 3.2.

Corollary 3.3. Suppose a pseudo-Riemannian space $V_{n}$ has a supplementary tensor which is skew-symmetric with respect to the first pair of indices and invariant $B=0$. Then the conditions (3.7) also hold in this space.

Another condition, which is true for Riemann tensor of a pseudo-Riemannian space, is Walker's identity:

$$
R_{h i j k,[m]}+R_{j k l m,[h i]}+R_{l m h i,[j k]}=0,
$$

where brackets $[i j]$ indicate an operation of alternating.

When we shift our attention back to a supplementary tensor, then similar conditions can be written down as follows

$$
\begin{aligned}
D_{h i j k,[l m]}+D_{j k l m,[h i]}+D_{l m h i,[j k]}=g_{h k} \varphi_{i j,[l m]}-g_{h j} \varphi_{i k .[l m]}+ \\
+g_{j m} \varphi_{k l,[h i]}-g_{j l} \varphi_{k m,[h i]}+g_{l i} \varphi_{m h,[j k]}-g_{l h} \varphi_{m i,[j k]} .
\end{aligned}
$$


We claim that the Walker's identity holds for a supplementary tensor whenever

$$
D_{h i j k,[m]}+D_{j k l m,[h i]}+D_{l m h i,[j k]}=0 .
$$

Indeed, otherwise, taking into account (3.12) and Ricci identity

$$
\begin{aligned}
& g_{h k}\left(\varphi_{\alpha i} R_{j l m}^{\alpha}+\varphi_{\alpha j} R_{i l m}^{\alpha}\right)-g_{h j}\left(\varphi_{i \alpha} R_{k l m}^{\alpha}+\varphi_{k \alpha} R_{i l m}^{\alpha}\right)+ \\
& \quad+g_{j m}\left(\varphi_{\alpha k} R_{l h i}^{\alpha}+\varphi_{\alpha l} R_{k h i}^{\alpha}\right)-g_{j l}\left(\varphi_{\alpha k} R_{m h i}^{\alpha}+\varphi_{\alpha m} R_{k h i}^{\alpha}\right)+ \\
& \quad+g_{l i}\left(\varphi_{\alpha m} R_{h j k}^{\alpha}+\varphi_{\alpha h} R_{m j k}^{\alpha}\right)-g_{l h}\left(\varphi_{\alpha m} R_{i j k}^{\alpha}+\varphi_{\alpha i} R_{m j k}^{\alpha}\right)=0 .
\end{aligned}
$$

Wrapping by indices $h$ and $k$, we arrive at

$$
\begin{gathered}
(n-1)\left(\varphi_{\alpha i} R_{j l m}^{\alpha}+\varphi_{\alpha j} R_{i l m}^{\alpha}\right)-g_{j m}\left(\varphi_{\alpha \beta} R_{. l i .}^{\alpha \beta}-\varphi_{\alpha l} R_{i}^{\alpha}\right)+ \\
+g_{j l}\left(\varphi_{\alpha \beta} R_{. m i .}^{\alpha \beta}-\varphi_{\alpha m} R_{i}^{\alpha}\right)+g_{l i}\left(\varphi_{\alpha \beta} R_{. m j .}^{\alpha}{ }^{\beta}-\varphi_{\alpha m} R_{j}^{\alpha}\right)- \\
-\left(\varphi_{\alpha m} R_{i j l}^{\alpha}+\varphi_{\alpha i} R_{m j l}^{\alpha}\right)=0 .
\end{gathered}
$$

Wrapping by indices $m$ and $i$, we can see that

$$
\varphi_{\alpha \beta} R_{. i j .}^{\alpha \beta}-\varphi_{\alpha i} R_{j}^{\alpha}=0
$$

and (3.14) can be written as follows:

$$
(n-1)\left(\varphi_{\alpha i} R_{j l m}^{\alpha}+\varphi_{\alpha j} R_{i l m}^{\alpha}\right)-\left(\varphi_{\alpha m} R_{i j l}^{\alpha}+\varphi_{\alpha i} R_{m j l}^{\alpha}\right)=0 .
$$

The latter implies that

$$
\varphi_{\alpha i} R_{j l m}^{\alpha}+\varphi_{\alpha j} R_{i l m}^{\alpha}=0 .
$$

On the other hand, if conditions (3.15) hold, then (3.12) implies (3.13). Thus, we proved the following theorem:

Theorem 3.4. The Walker's identity is true for a supplementary tensor $D_{i j k}^{h}$ if and only if the tensor $\varphi_{i j}$ complies to the condition (3.15).

Substituting (3.5) into (3.15) we get

Corollary 3.5. If a supplementary tensor $D_{i j k}^{h}$ is skew-symmetric with respect to the first pair of indices, then it complies to the Walker's identity.

Moreover, the following theorems are true:

Theorem 3.6. If a supplementary tensor $D_{i j k}^{h}$ complies to the condition (3.7), then the pseudo-Riemannian space is equidistant.

Theorem 3.7. If a supplementary tensor $D_{i j k}^{h}$ complies to the Walker's identity, then the pseudo-Riemannian space is equidistant. 


\section{Pseudo-Riemannian spaces With differential Conditions IMPOSED ON THE SUPPLEMENTARY TENSOR}

A pseudo-Riemannian space satisfying the following expression

$$
R_{i j k, l}^{h}=0,
$$

is called a symmetric space.

If a supplementary tensor is covariantly constant, i.e.

$$
D_{i j k, l}^{h}=0,
$$

then the Riemann tensor $\bar{V}_{n}$ is covariantly constant with respect to a connection of $V_{n}$.

Equation (4.2), together with (3.2), implies that

$$
R_{i j k, l}^{h}=\delta_{j}^{h} \varphi_{i k, l}-\delta_{k}^{h} \varphi_{i j, l} .
$$

Let us lower down index $h$ with the help of the metric tensor of $V_{n}$ and symmetrize by indices $h$ and $i$ :

$$
g_{h j} \varphi_{i k, l}-g_{h k} \varphi_{i j, l}+g_{i j} \varphi_{h k, l}-g_{i k} \varphi_{h j, l}=0 .
$$

Wrapping by indices $h$ and $j$ we get

$$
n \cdot \varphi_{i k, l}=\tau_{, l} g_{i k},
$$

where $\tau_{, l}=\varphi_{\alpha ., l}^{\alpha}=\varphi_{\alpha \beta, l} g^{\alpha \beta}$.

Wrapping (4.3) by indices $h$ and $k$, we obtain

$$
R_{i j, l}=(1-n) \varphi_{i j, l} .
$$

Condition (4.5) is true for pseudo-Riemannian spaces, which admits $\varphi($ Ric) vector fields [4,5]. Geodesic mappings of these spaces are studied in [27]. Also [2] treated spaces with generalized $\varphi($ Ric $)$ vector fields.

Substituting (4.4) into (4.3), we obtain

$$
R_{i j k, l}^{h}=\frac{\tau_{, l}}{n}\left(\delta_{j}^{h} g_{i k}-\delta_{k}^{h} g_{i j}\right) .
$$

Cycling (4.6) by indices $j, k, l$ gives

$$
\tau_{, l}\left(\delta_{j}^{h} g_{i k}-\delta_{k}^{h} g_{i j}\right)+\tau_{, j}\left(\delta_{k}^{h} g_{i l}-\delta_{l}^{h} g_{i k}\right)+\tau_{, k}\left(\delta_{l}^{h} g_{i j}-\delta_{j}^{h} g_{i l}\right)=0,
$$

and wrapping it by $h$ and $j$ we get

$$
\tau_{, l} g_{i k}-\tau_{, k} g_{i l}=0 .
$$

The latter implies:

$$
\tau_{, l}=0 \text {. }
$$

Thus, (4.6) is transformed to (4.1), and we see that a pseudo-Riemannian space, including covariantly constant supplementary tensor, is a symmetric 
space. Symmetric spaces admit non-trivial geodesic mappings only when they are spaces of constant curvature, [24].

Theorem 4.1. Covariantly constant tensor exists only in spaces of constant curvature.

Let us treat pseudo-Riemannian spaces, in which where the following expression holds:

$$
D_{i j k, l_{1} l_{2} \cdots l_{m}}^{h}=0, \quad m=\overline{1, m} .
$$

Then the following theorem holds:

Theorem 4.2. If a supplementary tensor complies to the condition (4.7), then the following conditions are true for the Riemann tensor:

$$
R_{i j k, l_{1} l_{2} \cdots l_{m}}^{h}=0, \quad m=\overline{1, m} .
$$

The research on geodesic mappings of spaces, where the conditions (4.8) are true, is carried out for $m=1,2$ in [19,20], for $m=3$ in [21], and finally for $m \geqslant 3$, under additional assumption of semi-symmetry, in [25].

A pseudo-Riemannian space $V_{n}$ whose Riemann tensor complies to the following condition

$$
R_{i j k,[l m]}^{h}=0
$$

is called semi-symmetric, [24], while (4.9) is called in turn a condition of semi-symmetry.

Consider a pseudo-Riemannian space $V_{n}$ with a supplementary tensor complying to the condition of semi-symmetry, namely

$$
D_{i j k,[m]}^{h}=0 .
$$

Taking into account (3.2), we obtain

$$
R_{i j k, l m]}^{h}=\delta_{j}^{h} \varphi_{i k,[l m]}-\delta_{k}^{h} \varphi_{i j,[l m]} .
$$

However, note that (4.10) implies that the Walker's conditions are true for a supplementary tensor of a given pseudo-Riemannian space. Hence

$$
\varphi_{i j,[m]}=0 .
$$

Thus, we get the following

Theorem 4.3. If a supplementary tensor is semi-symmetric, then the pseudo-Riemannian space $V_{n}$ is semi-symmetric as well.

Semi-symmetric spaces distinct from spaces of constant curvature admit non-trivial geodesic mappings only when they are equidistant. 
It is well-known, that equidistant pseudo-Riemannian spaces with an equidistant vector field, which is not covariantly constant, always admit non-trivial geodesic mappings.

\section{Proofs of Theorems}

Proof for theorem 3.6. Integrability conditions for equations (2.5) can be written down as follows

$$
a_{\alpha i} R_{j k l}^{\alpha}+a_{\alpha j} R_{i k l}^{\alpha}=\lambda_{l, i} g_{j k}+\lambda_{l, j} g_{i k}-\lambda_{k, i} g_{j l}-\lambda_{k, j} g_{i l} .
$$

Let us multiply this relation by vector $\varphi^{l}=g^{\alpha l} \varphi_{\alpha}$ and wrap it by index $l$. Then taking into account (3.7) we obtain

$$
\varphi^{\alpha} \lambda_{l, i} g_{j k}+\varphi^{\alpha} \lambda_{l, j} g_{i k}-\varphi_{j} \lambda_{k, i}-\varphi_{i} \lambda_{k, j}=0 .
$$

Wrapping further by indices $j$ and $k$ we get

$$
\varphi^{\alpha} \lambda_{l, i}=\frac{\mu}{n} \varphi_{i} .
$$

Substitute now (5.3) into (5.2) and group it

$$
\varphi_{j}\left(\lambda_{k, i}-\frac{\mu}{n} g_{k i}\right)+\varphi_{i}\left(\lambda_{k, j}-\frac{\mu}{n} g_{k j}\right)=0 .
$$

Alternating by indices $k$ and $j$ we obtain

$$
\varphi_{j}\left(\lambda_{k, i}-\frac{\mu}{n} g_{k i}\right)-\varphi_{k}\left(\lambda_{j, i}-\frac{\mu}{n} g_{j i}\right)=0 .
$$

Re-designate indices $k$ and $i$

$$
\varphi_{j}\left(\lambda_{k, i}-\frac{\mu}{n} g_{k i}\right)-\varphi_{i}\left(\lambda_{j, k}-\frac{\mu}{n} g_{j k}\right)=0 .
$$

and add up (5.5) and (5.4):

$$
\varphi_{j}\left(\lambda_{k, i}-\frac{\mu}{n} g_{k i}\right)=0 .
$$

Since $\varphi_{j} \not \equiv 0$,

$$
\lambda_{k, i}=\frac{\mu}{n} g_{k i} .
$$

This proves Theorem 3.6. 
Proof of Theorem 3.7. We will apply methods developed in [9, 10, 20].

Multiply (5.1) by $\varphi_{m}^{l}=\varphi_{m \alpha} g^{\alpha l}$. Wrapping further by index $l$, symmetrizing the latter by indices $k$ and $m$, and taking into account (3.15), we obtain

$$
\begin{aligned}
& \varphi_{m}^{\alpha} \lambda_{\alpha, i} g_{j k}+\varphi_{m}^{\alpha} \lambda_{\alpha, j} g_{i k}-\lambda_{k, i} \varphi_{j m}-\lambda_{k, j} \varphi_{i m}+ \\
& \quad+\varphi_{k}^{\alpha} \lambda_{\alpha, i} g_{j m}+\varphi_{k}^{\alpha} \lambda_{\alpha, j} g_{i m}-\lambda_{m, i} \varphi_{j k}-\lambda_{m, j} \varphi_{i k}=0 .
\end{aligned}
$$

Wrapping by indices $j$ and $k$ gives

$$
(n+1) \varphi_{m}^{\alpha} \lambda_{\alpha i}-\mu \varphi_{i m}+\varphi^{\alpha \beta} \lambda_{\alpha, \beta} g_{i m}-\varphi_{\alpha}{ }^{\alpha} \lambda_{m, i}-\lambda_{m, \alpha} \varphi_{i}^{\alpha}=0 .
$$

Alternating the latter, we see that

$$
\varphi_{m}^{\alpha} \lambda_{\alpha i}=\varphi_{i}^{\alpha} \lambda_{m, \alpha} .
$$

Hence (5.7) can be written down as follows

$$
\varphi_{m}^{\alpha} \lambda_{\alpha i}=\stackrel{1}{\tau} g_{i m}+\stackrel{2}{\tau} \lambda_{i m}+\stackrel{3}{\tau} \varphi_{i m},
$$

where

$$
\stackrel{1}{\tau}=-\frac{\varphi^{\alpha \beta} \lambda_{\alpha, \beta}}{n}, \quad \quad \stackrel{2}{\tau}=\frac{\varphi_{\alpha}^{\alpha} .}{n}, \quad \stackrel{3}{\tau}=\frac{\mu}{n} .
$$

Alternating (5.6) by indices $i$ and $k$ we get

$$
\begin{aligned}
& \varphi_{m}^{\alpha} \lambda_{\alpha, i} g_{j k}-\varphi_{m}^{\alpha} \lambda_{\alpha, k} g_{j i}-\lambda_{k, j} \varphi_{i m}+\lambda_{i, j} \varphi_{k m}+ \\
& \quad+\varphi_{k}^{\alpha} \lambda_{\alpha, j} g_{i m}-\varphi_{i}^{\alpha} \lambda_{\alpha, j} g_{k m}-\lambda_{m, i} \varphi_{j k}+\lambda_{m, k} \varphi_{j i}=0 .
\end{aligned}
$$

Let us re-designate indices $k$ and $j$

$$
\begin{aligned}
& \varphi_{m}^{\alpha} \lambda_{\alpha, i} g_{k j}-\varphi_{m}^{\alpha} \lambda_{\alpha, j} g_{k i}-\lambda_{k, j} \varphi_{i m}+\lambda_{i, k} \varphi_{j m}+ \\
& \quad+\varphi_{k}^{\alpha} \lambda_{\alpha, j} g_{i m}-\varphi_{i}^{\alpha} \lambda_{\alpha, k} g_{j m}-\lambda_{m, i} \varphi_{k j}+\lambda_{m, j} \varphi_{k i}=0 .
\end{aligned}
$$

Adding up equations (5.9) and (5.6) we obtain

$$
\varphi_{m}^{\alpha} \lambda_{\alpha, i} g_{j k}+\varphi_{k}^{\alpha} \lambda_{\alpha, j} g_{i m}-\lambda_{k, j} \varphi_{i m}-\lambda_{m, i} \varphi_{k j}=0 .
$$

Substituting further (5.8) into (5.10) gives

$$
\begin{aligned}
2 \stackrel{1}{\tau} g_{m i} g_{j k}+\stackrel{2}{\tau} & \lambda_{m, i} g_{j k}+\stackrel{2}{\tau} \lambda_{k, j} g_{i m}+ \\
& +\stackrel{3}{\tau} \varphi_{i m} g_{j k}+\stackrel{3}{\tau} \varphi_{k j} g_{i m}-\lambda_{k, j} \varphi_{i m}-\lambda_{m, i} \varphi_{k j}=0 .
\end{aligned}
$$

Let us group the latter as follows:

$$
\left(\lambda_{m, i}-\stackrel{3}{\tau} g_{m i}\right)\left(\varphi_{j k}-\stackrel{2}{\tau} g_{j k}\right)+\left(\lambda_{j, k}-\stackrel{3}{\tau} g_{j k}\right)\left(\varphi_{m i}-\stackrel{2}{\tau} g_{m i}\right)=0
$$

The latter implies that $\lambda_{m, i}=\stackrel{3}{\tau} g_{m i}$, or $\varphi_{m i}=\stackrel{2}{\tau} g_{m i}$. Theorem 3.7 is completed. 
Proof of Theorem 4.2. Equation (4.7) together with (3.2) implies

$$
R_{i j k, l_{1} l_{2} \cdots l_{m}}^{h}+\delta_{k}^{h} \varphi_{i j, l_{1} l_{2} \cdots l_{m}}-\delta_{j}^{h} \varphi_{i k, l_{1} l_{2} \cdots l_{m}}=0 .
$$

Let us lower down index $h$ and alternate it by indices $h$ and $i$. Then

$$
g_{h k} \varphi_{i j, l_{1} l_{2} \cdots l_{m}}+g_{i k} \varphi_{h j, l_{1} l_{2} \cdots l_{m}}-g_{h j} \varphi_{i k, l_{1} l_{2} \cdots l_{m}}-g_{i j} \varphi_{h k, l_{1} l_{2} \cdots l_{m}}=0 .
$$

Wrapping by indices $h$ and $k$ we get

$$
\varphi_{i j, l_{1} l_{2} \cdots l_{m}}=\frac{1}{n} \varphi_{\alpha ., l_{1} l_{2} \cdots l_{m}}^{\alpha} g_{i j} .
$$

Substituting the latter into (5.11) we obtain

$$
R_{i j k, l_{1} l_{2} \cdots l_{m}}^{h}+\frac{1}{n} \varphi_{\alpha ., l_{1} l_{2} \cdots l_{m}}^{\alpha}\left(\delta_{k}^{h} g_{i j}-\delta_{j}^{h} g_{i k}\right)=0 .
$$

Cycling by indices $j, k, l$ gives

$$
\begin{aligned}
\left(\delta_{k}^{h} g_{i j}-\delta_{j}^{h} g_{i k}\right) \varphi_{\alpha \cdot, l_{1} l_{2} \cdots l_{m}}^{\alpha}+\left(\delta_{l_{1}}^{h} g_{i k}\right. & \left.-\delta_{k}^{h} g_{i l_{1}}\right) \varphi_{\alpha \cdot, j l_{2} \cdots l_{m}}^{\alpha}+ \\
& +\left(\delta_{j}^{h} g_{i l_{1}}-\delta_{l_{1}}^{h} g_{i j}\right) \varphi_{\alpha \cdot, k l_{2} \cdots l_{m}}^{\alpha}=0 .
\end{aligned}
$$

Wrapping by indices $h$ and $k$ we get

$$
(n-2)\left(g_{i j} \varphi_{\alpha,{ }_{1} l_{2} \cdots l_{m}}^{\alpha}-g_{i l_{1}} \varphi_{\alpha ., j l_{2} \cdots l_{m}}^{\alpha}\right)=0,
$$

which implies

$$
\varphi_{\alpha ., l_{1} l_{2} \cdots l_{m}}^{\alpha}=0 .
$$

Finally, substituting it into (5.11), we see that Theorem 4.2 is true.

\section{Conclusions}

In order to facilitate the research on the geodesic mappings of pseudoRiemannian spaces we apply a special supplementary tensor. By definition, the supplementary tensor equals to Riemann tensor of the pseudoRiemannian space, which is in geodesic correspondence to a given space $V_{n}$. The research is carried out in the space $V_{n}$, while the conditions are imposed on the Riemann tensor of $\bar{V}_{n}$. Those conditions are usually used for specialization of spaces. However lowering down of the indices is defined by a metric tensor of $V_{n}$, while covariant derivative is calculated by a connection of $V_{n},[13-15]$.

In all the cases we lead to equidistant spaces. Equidistant spaces are closed in relation to non-trivial geodesic mappings. The application of a supplementary tensor allows to study simultaneously the properties of a pair of pseudo-Riemannian spaces in geodesic correspondence, $[7,16,18]$.

The developed methods can be applied for study of holomorphic-projective mappings of Kählerian spaces and other mappings of generalized spaces, $[12,17]$. The obtained results can find an application in the general relativity theory and mechanics, [3]. 


\section{REFERENCES}

[1] A. V. Bolsinov, V. A. Kiosak, V. S. Matveev. A Fubini theorem for pseudoRiemannian geodesically equivalent metrics. J. Lond. Math. Soc., 80(2):341-356, 2009, doi: $10.1112 / \mathrm{jlms} / \mathrm{jdp} 032$.

[2] K. De, U. C. De. Almost quasi-yamabe solitons and gradient almost quasiyamabe solitons in paracontact geometry. Quaestiones Mathematicae, 2020, doi: $10.2989 / 16073606.2020 .1799882$.

[3] D. Doikov, V. A. Kiosak. On the Schwarzschild model for gravitating objects of the universe. AIP Conference Proceedings, 2302(040001), 2020, doi: 10.1063/5.0033657.

[4] I. Hinterleitner, V. A. Kiosak. $\varphi$ (Ric)-vector fields in Riemannian spaces. Archivummathematicum, 44(5):385-390, 2008.

[5] I. Hinterleitner, V. A. Kiosak. $\varphi$ (Ric)-vector fields on conformally flat spaces. Proceedings of American Institute of Physics, 1191:98-103, 2009, doi: 10.1063/1.3275604.

[6] D. S. Kalyuzhniy. Multiparametric dissipative linear stationary dynamical scattering systems: discrete case. J. Operator Theory, 43(2):427-460, 2000.

[7] V. A. Kiosak, G. V. Kovalova. Geodesic mappings of quasi-Einstein spaces with a constant scalar curvature. Matematychni Studii, 53(2):212-217, 2020, doi: $10.30970 / \mathrm{ms} .53 .2 .212-217$.

[8] V. A. Kiosak, V. S. Matveev. Complete Einstein metrics are geodesically rigid. Comm. Math. Phys., 289(1):383-400, 2009, doi: 10.1007/s00220-008-0719-7.

[9] V. A. Kiosak, V. S. Matveev. Proof of the projective Lichnerowicz conjecture for pseudo-Riemannian metrics with degree of mobility greater than two. Comm. Math. Phys., 297(2):401-426, 2010, doi: 10.1007/s00220-010-1037-4.

[10] V. A. Kiosak, V. S. Matveev. There exist no 4-dimensional geodesically equivalent metrics with the same stress-energy tensor. Journal of Geometry and Physics, 78:1-11, 2014, doi: 10.1016/j.geomphys.2014.01.002.

[11] V. A. Kiosak, V. S. Matveev, J. Mikes, I. G. Shandra. On the degree of geodesic mobility for Riemannian metrics. Mathematical Notes, 87(3-4):586-587, 2010, doi: $10.1134 /$ S0001434610030375.

[12] V. A. Kiosak, A. G. Savchenko, O. Gudyreva. On the conformal mappings of special quasi-Einstein spaces. AIP Conference Proceedings, 2164(040001), 2019, doi: $10.1063 / 1.5130793$.

[13] V. A. Kiosak, A. G. Savchenko, A. Kamienieva. Geodesic mappings of compact quasi-Einstein spaces with constant scalar curvature. AIP Conference Proceedings, 2302(040002), 2020, doi: 10.1063/5.0033661.

[14] V. A. Kiosak, A. G. Savchenko, S. Khniunin. On the typology of quasi-Einstein spaces. AIP Conference Proceedings, 2302(040003), 2020, doi: 10.1063/5.0033700.

[15] V. A. Kiosak, A. G. Savchenko, G. V. Kovalova. Geodesic mappings of compact quasiEinstein spaces, 1. Proceedings of the International Geometry Center, 13(1):35-48, 2020, doi: $10.15673 /$ tmgc.v13i1.1711.

[16] V. A. Kiosak, A. G. Savchenko, O. Latysh. Geodesic mappings of compact quasiEinstein spaces, II. Proceedings of the International Geometry Center, 14(1):81-92, 2021, doi: 10.15673/tmgc.v14i1.1936.

[17] V. A. Kiosak, A. G. Savchenko, T. Shevchenko. Holomorphically projective mappings of special Kähler manifolds. AIP Conference Proceedings, 2025(080004), 2018, doi: $10.1063 / 1.5064924$. 
[18] J. Mikes, I. Hinterleitner, V.A. Kiosak. On the theory of geodesic mappings of Einstein spaces and their generalizations. AIP Conference Proceedings, 861:428-435, 2006, doi: $10.1063 / 1.2399606$.

[19] J. Mikesh. Geodesic Ricci mappings of two-symmetric Riemann spaces. Math. Notes, 28(2):622-624, 1980, doi: 10.1007/bf01157926.

[20] J. Mikesh. Geodesic mappings of m-symmetric and generaliz semisymmetric spaces. Russian Math. (Iz. VUZ), 36(8):38-42, 1992.

[21] J. Mikesh, V. S. Sobchuk. Geodesic mappings of 3-symmetric Riemannian spaces. J. Math. Sci., 69(1):885-887, 1994, doi: 10.1007/BF01250819.

[22] A. O. Prishlyak, M. V. Loseva. Optimal Morse-Smale flows with singularities on the boundary of a surface. J Math Sci, 243:279-286, 2019, doi: 10.1007/s10958-019-04539-9.

[23] A. O. Prishlyak, M. V. Loseva. Topology of optimal flows with collective dynamics on closed orientable surfaces. Proc. Int. Geom. Cent., 13(2):50-67, 2020, doi: 10.15673/tmgc.v13i2.1731.

[24] N. S. Sinyukov. Geodesic mappings of Riemannian spaces. Nauka, Moscow, 1979.

[25] V. S. Sobchuk. Geodesic mappings of some classes of Riemannian spaces. Soviet Math. (Iz. VUZ), 34(4):56-59, 1990.

[26] Cornelis van der Mee, V. Pivovarchik. Inverse scattering for a Schrödinger equation with energy dependent potential. Journal of Mathematical Physics, 42(1):158-181, 2001, doi: 10.1063/1.1326921.

[27] Y. Vashpanov, O. Olshevska, O. Lesechko. Geodesic mappings of spaces with $\varphi($ Ric) vector fields. AIP Conference Proceedings, 2302(040010), 2020, doi: 10.1063/5.0033965.

[28] M. M. Zarichnyi, A. G. Savchenko, V. A. Kiosak. Strong topology on the set of persistence diagrams. AIP Conference Proceedings, 2164(040006), 2019, doi: $10.1063 / 1.5130798$.

Received: November 4, 2021, accepted: November 29, 2021.

V. Kiosak

Odesa State Academy of Civil Engineering and Architecture, Didrihson st., 4, Odesa, 65029, UKRAINE

Email: kiosakv@ukr.net

ORCID: 0000-0002-7433-6709

O. Prishlyak

Taras Shevchenko National University of Kyiv, 4th Academician Glushkov avEnUe, Kyiv, 03127, UkRAine

Email: prishlyak@yahoo.com

ORCID: 0000-0002-7164-807X

O. Lesechko

Odesa State Academy of Civil Engineering and Architecture, Didrihson st., 4, Odesa, 65029, Ukraine

Email: a.lesechko@ukr.net

ORCID: 0000-0002-2352-6174 